\title{
Sociobiology
}

RESEARCH ARTICLE - ANTS

\section{Insecticidal effect of volatile compounds from plant materials of Murraya exotica against Red Imported Fire Ant Workers}

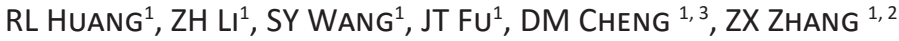 \\ 1 - South China Agricultural University, Guangzhou, China \\ 2 - State Key Laboratory for Conservation and Utilization of Subtropical Agro-Bioresources, Guangzhou, China \\ 3 - Zhongkai University of Agriculture and Engineering, Guangzhou, China
}

\author{
Article History \\ Edited by \\ Jacques H. C. Delabie, CEPLAC, Brazil \\ Received 17 December 2015 \\ Initial acceptance 21 April 2016 \\ Final acceptance 09 May 2016 \\ Publication date 15 July 2016 \\ Keywords \\ Volatile compounds, Murraya exotica, \\ Solenopsis invicta, activity.

\section{Corresponding author} \\ Zhixiang Zhang, \\ Key Laboratory of Natural Pesticide and \\ Chemical Biology \\ Ministry of Education South China \\ Agricultural University \\ 510642, Guangzhou, China \\ E-Mail: zdsys@scau.edu.cn
}

\begin{abstract}
The effect of volatile compounds from the mashed fresh, fallen, and dried leaves of Murraya exotica on the behavior of red imported fire ant (Solenopsis invicta, RIFA) workers was investigated by fumigation toxicity bioassay. The volatile compounds from different mashed leaves (fresh, fallen, and dried leaves) of $M$. exotica were collected by solidphase microextraction and identified by gas chromatography-mass spectrometry. $\beta$-caryophyllene, $\alpha$-cedrene, $\alpha$-copaene, $\beta$-cubebene, and germacrene $D$ were identified as major components of the volatile compounds. In exposure time from $1 \mathrm{~d}$ to $9 \mathrm{~d}$, the mortality of RIFA increased from $5.00 \%$ to $100.00 \%$ (fresh leaves), $11.67 \%$ to $93.33 \%$ (fallen leaves), and $15.00 \%$ to $83.33 \%$ (dried leaves) in minor workers, whereas in major workers, the increases were from $13.33 \%$ to $93.33 \%$ (fresh leaves), $6.67 \%$ to $83.33 \%$ (fallen leaves), and $10.00 \%$ to $60.00 \%$ (dried leaves). The volatile compounds reduced the walking and grasping abilities and aggregation rate of RIFA workers. Results indicate that mashed leaves of $M$. exotica have potential for controlling RIFA.
\end{abstract}

\section{Introduction}

Red imported fire ant (Solenopsis invicta Buren 1972 (Myrmicinae), RIFA), native from Parana River basin of South America, is a voracious consumer of numerous dead animals, such as arthropods, earthworms and vertebrates (Nattrass \& Vanderwoude, 2001). RIFAs have become a pest in southern United States, Australia, New Zealand, Thailand, Taiwan, and the Philippines, and they were introduced to southern Chinese Provinces of Guangdong, Guangxi, and Fujian in 2005 (Ascunce et al., 2011; Zhang et al., 2007). In several highly infested areas, RIFAs have caused the decline of native ant species by up to $90 \%$ through displacement (Porter \& Savignano, 1990). RIFAs have caused severe damage to human, animals, agriculture, and environment. Furthermore, these ants negatively affect the local biodiversity and cause approximately US $\$ 5$ billion losses yearly in urban and agricultural areas in the United States (Cheng et al., 2008). Traditional methods such as insecticides and baits are used for managing S. invicta; however, these methods cause pollution to the environment. Therefore, new methods such as natural and environment-friendly insecticides should be found to control RIFAs (Vogt et al., 2002; Appel et al., 2004).

Murraya exotica belongs to the family Rutaceae and is an evergreen shrub or occasionally a small tree, which is mainly distributed in the west and southeast of China. Its flowers are few, white and fragrant (Zhang et al., 2008). Previous studies have reported the chemical compositions of essential oils from flowers, leaves, and stems of $M$. exotica (Pino et al., 2006; Raina et al., 2006; Rout et al., 2007; Olawore et al., 
2005; Negi et al., 2005). Yu et al. (2001) reported that $M$. exotica extract on some fungi has significant inhibitory effect. Li et al. (2001) found the essential oils of M. exotica have good control effect on stored grain pests. Moreover, Luo et al. (2005) found that the extracts of M. exotica indicated very strong antifeedant activities against the third instar and fifth instar larvae. However, no research has yet been conducted on the insecticidal activity of the essential oil of $M$. exotica against RIFAs. This study is the first to report on the toxicity of the volatile compounds released from the mashed leaves of M. exotica against RIFAs. Moreover, the present study investigates the effects of the volatile compounds from the mashed leaves of M. exotica on the RIFA workers.

\section{Materials and methods}

\section{Plant materials}

The leaves of $M$. exotica were collected from the Insecticidal Botanical Garden at the South China Agricultural University. Three different leaves of M. exotica were used for the test. The fresh leaves were cut off and immediately sent to the laboratory. The fallen leaves were collected from the ground below M. exotica. The dried leaves were gained by putting the fresh leaves in baking box with $40^{\circ} \mathrm{C}$ for $5 \mathrm{~h}$.

\section{Insects}

S. invicta colonies were obtained from a suburb in Guangzhou and stored in the laboratory for bioassays in plastic containers at $25 \pm 2{ }^{\circ} \mathrm{C}$ and $60 \%$ to $80 \%$ RH. A test tube $(25 \mathrm{~mm} \times 200 \mathrm{~mm})$ partially filled with $10 \%$ honey water and plugged with cotton was used as a water source, and a Petri dish $(8.5 \mathrm{~cm} \times 1.5 \mathrm{~cm})$ containing the larvae of Tenebrio molitor L. (Coleoptera Tenebrionidae) was used as a food source. RIFAs were kept in a dry indoor environment at $25 \pm$ $2{ }^{\circ} \mathrm{C}$ until the experiment was over.

\section{Fumigation toxicity bioassay}

Fresh, fallen, and dried leaves of $M$. exotica were crushed thoroughly in a blender for $5 \mathrm{~min}$. About $30 \mathrm{~g}$ of mashed leaves was placed on the bottom of a $1000 \mathrm{ml}$ breaker. Twenty minor workers (body length $=2.6 \mathrm{~mm}$ to 3.0 $\mathrm{mm}$, head width $=0.5 \mathrm{~mm}$ to $0.6 \mathrm{~mm}$ ) and 10 major workers (body length $=4.2 \mathrm{~mm}$ to $4.6 \mathrm{~mm}$, head width $=1.0 \mathrm{~mm}$ to $1.2 \mathrm{~mm}$ ) were placed on the bottom of a $100 \mathrm{ml}$ breaker. The breaker was coated with Fluon emulsion inside vertical wall to prevent the ants from escaping and placed on the bottom of a $1000 \mathrm{ml}$ breaker without covering the mashed leaves. The $1000 \mathrm{ml}$ beaker was covered with plastic film. The ants were placed on the laboratory maintained at $25 \pm 2{ }^{\circ} \mathrm{C}$ and $65 \% \pm 5 \%$ relative humidity. All treatments were replicated thrice. The contrast was the absence of mashed leaves in the $1000 \mathrm{ml}$ beaker.
Physiological index observation of RIFA workers

The mortalities of the workers were observed 1, 3, 5,7 , and $9 \mathrm{~d}$ after adding the $100 \mathrm{ml}$ beaker to the leaves. Behavioral observation on the grasping ability of the workers was determined $1,3,5,7$, and $9 \mathrm{~d}$ after adding the $100 \mathrm{ml}$ beaker to the leaves. The workers were placed on an A4 paper (made from plant fibre, $210 \mathrm{~mm} \times 297 \mathrm{~mm}$ ), which was slowly turned over to 180 degrees for $1 \mathrm{~min}$. If the ants would not fall down from the A4 paper, they were regarded as possessing grasping ability.

The formula was as follows: grasping rate $=$ number of worker ants possessing grasping ability/number of worker ants per replicate $\times 100$.

Behavioral observation on the walking ability of the workers was determined $1,3,5,7$, and $9 \mathrm{~d}$ after testing. The workers were placed on an A4 paper. The ants were regarded as possessing walking ability if they could walk continuously for $10 \mathrm{~cm}$ without falling down from the A4 paper.

The formula was as follows: walking rate $=$ number of worker ants possessing walking ability/number of worker ants per replicate $\times 100$.

Behavioral observation on the aggregation of the workers was determined $1,3,5,7$, and $9 \mathrm{~d}$ after testing. The aggregating level based on the method employed by Depickere et al. (2004) and Devigne et al. (2011). The workers were regarded as aggregating if over two workers gathered, and the distance between each other was less than $0.5 \mathrm{~cm}$.

The formula was as follows: aggregation rate $=$ number of worker ants in aggregate mass/number of worker ants per replicate $\times 100$.

\section{Extraction of volatiles by solid-phase microextraction}

Fresh, fallen, and dried leaves were mashed with a high-speed organization stamp mill for $5 \mathrm{~min}$, and then $30 \mathrm{~g}$ of mashed leaves was placed into a $250 \mathrm{ml}$ glass Erlenmeyer flask covered with silver paper.

The manual solid-phase microextraction device equipped with a $100 \mu \mathrm{m}$ polydimethylsiloxane fiber (Supelco) was employed in this study. The fiber was activated at constant temperature $\left(250^{\circ} \mathrm{C}\right)$ for $30 \mathrm{~min}$ before use. The activated fiber was then pushed into the Erlenmeyer flask contained mashed leaves to absorb the volatiles for $40 \mathrm{~min}$. Fiber was then poured into the injection port of the gas chromatographymass spectrometry (GC-MS) with a temperature of $250^{\circ} \mathrm{C}$ for $3 \mathrm{~min}$, and the volatiles were analyzed by GC-MS.

\section{Chemical Analysis by Gas Chromatography-Mass spectrometry}

The sample was detected by an Agilent 6890 gas chromatograph equipped with an Agilent mass spectrometer detector. A DB- 5 capillary column $(30.00 \mathrm{~m} \times 0.25 \mathrm{~mm}$; film thickness by $0.25 \mathrm{~mm}$ ) was held at $50^{\circ} \mathrm{C}$ for $1 \mathrm{~min}$, raised to $200{ }^{\circ} \mathrm{C}$ at the rate of $3^{\circ} \mathrm{C} / \mathrm{min}$ for $2 \mathrm{~min}$, and raised to $230^{\circ} \mathrm{C}$ $\left(10^{\circ} \mathrm{C} / \mathrm{min}\right)$ for $2 \mathrm{~min}$. The injection temperature was set at 230 
${ }^{\circ} \mathrm{C}$. The detector was operated at $280^{\circ} \mathrm{C}$. Helium was used as a carrier gas at a flow rate of $1 \mathrm{ml} / \mathrm{min}$. The compounds were identified by retention times, Kovats indices, and mass spectra.

\section{Statistical Analysis}

Data were transformed into arcsine square root values for a three-way analysis of variance (ANOVA) to determine the significance of the effects of plant material, exposure time, mortalities, grasping rate, walking rate, and aggregation of the minor and major workers as well as various interactions. Furthermore, the differences in the data were assessed using Duncan's multiple range test, with $\mathrm{P}<0.05$ considered statistically significant. The figures were generated using Microsoft Office Excel 2007.

\section{Results}

\section{Results of ANOVA}

The mortalities, grasping rate, walking rate, and aggregation rate of workers may vary significantly according to plant material, exposure time, and worker size (ANOVA, $\mathrm{P}<0.05)$. The results reveal that the three main effects and the partial interactions are significant (Table 1). However, the mortalities, grasping rate, walking rate, and aggregation rate of RIFAs had no difference between with the interaction exposure time $\times$ worker size $(\mathrm{P}=0.2626,0.2544,0.2431$, $0.3612)$ and the interaction plant material $\times$ exposure time $\times$ worker size $(\mathrm{P}=0.1496,0.4848,0.4598,0.0922)$.

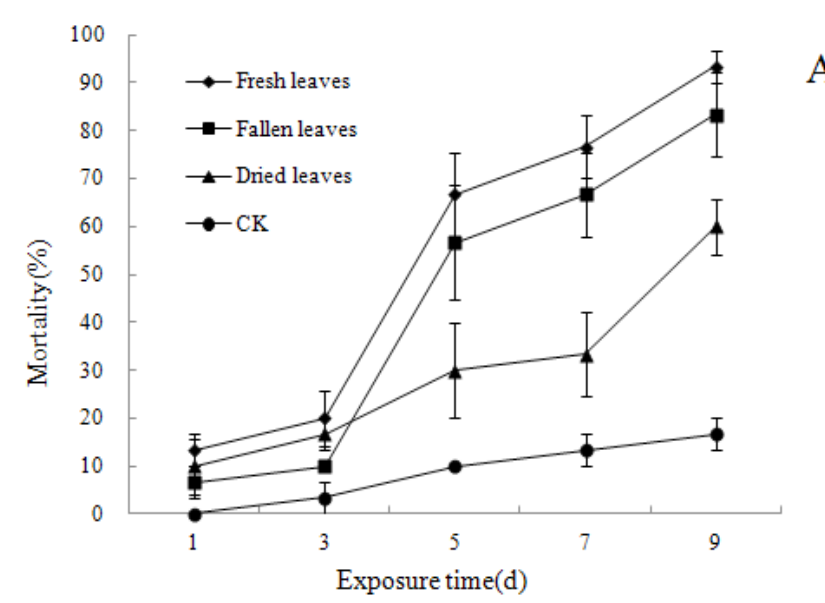

A

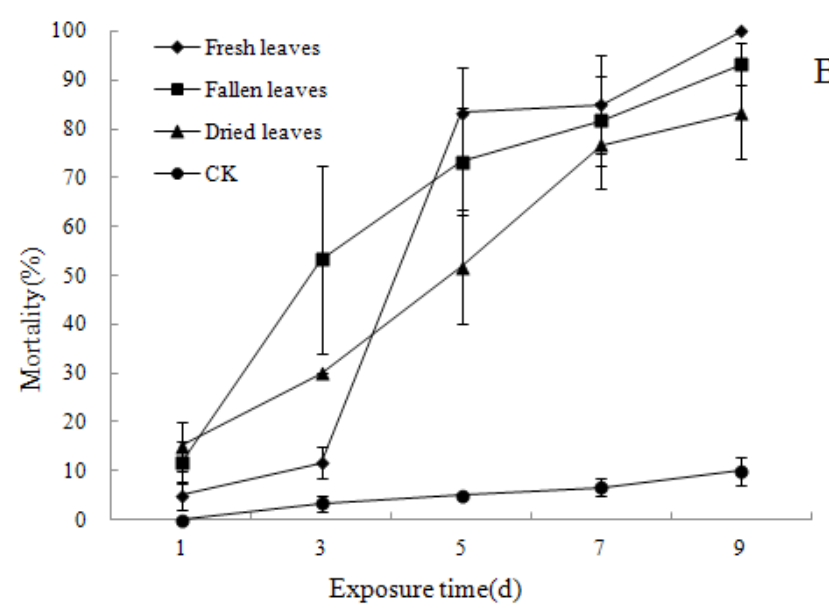

Fig 1. Mortalities of workers (A: major workers, B: minor workers) after treated with different mashed leaves of M. exotica.

Table 1. ANOVA for the main factors of the fumigation test affecting the behaviors of RIFA.

\begin{tabular}{|c|c|c|c|c|c|c|c|c|c|}
\hline \multirow{2}{*}{ Factors } & \multirow{2}{*}{$\mathrm{df}$} & \multirow{2}{*}{$\frac{\text { Mortality }}{\text { F values }}$} & \multicolumn{3}{|c|}{ Grasping rate } & \multicolumn{2}{|c|}{ Walking rate } & \multicolumn{2}{|c|}{ Aggregation rate } \\
\hline & & & $P$ values & $F$ values & $P$ values & F values & $P$ values & F values & $P$ values \\
\hline A & 3 & 105.002 & 0.0001 & 105.558 & 0.0001 & 107.198 & 0.0001 & 14.0164 & 0.0001 \\
\hline B & 4 & 104.588 & 0.0001 & 109.724 & 0.0001 & 113.959 & 0.0001 & 47.0154 & 0.0001 \\
\hline $\mathrm{C}$ & 1 & 19.3056 & 0.0001 & 16.8368 & 0.0001 & 17.2425 & 0.0001 & 43.0126 & 0.0001 \\
\hline $\mathrm{A} \times \mathrm{B}$ & 12 & 9.9821 & 0.0001 & 10.3586 & 0.0001 & 10.7487 & 0.0001 & 4.4143 & 0.0001 \\
\hline $\mathrm{A} \times \mathrm{C}$ & 3 & 7.3649 & 0.0002 & 6.3701 & 0.0006 & 6.9493 & 0.0003 & 0.3572 & 0.784 \\
\hline $\mathrm{B} \times \mathrm{C}$ & 4 & 1.3393 & 0.2626 & 1.3625 & 0.2544 & 1.3953 & 0.2431 & 1.1025 & 0.3612 \\
\hline $\mathrm{A} \times \mathrm{B} \times \mathrm{C}$ & 12 & 1.479 & 0.1496 & 0.9694 & 0.4848 & 0.9967 & 0.4598 & 1.6587 & 0.0922 \\
\hline
\end{tabular}

A: Plant material, B: Exposure time, $\mathrm{C}$ : Worker size $(\mathrm{P}=0.05)$

\section{Fumigation toxicity bioassay}

According to Fig 1, both major and minor workers exposed to volatiles from mashed leaves of M. exotica show increased mortality over time.

After $1 \mathrm{~d}$ exposure, the volatile compounds of fresh, fallen, and dried leaves of $M$. exotica lead to $5 \%, 11.6 \%$, and $10 \%$ mortality in minor workers as well as $13.33 \%$, $6.67 \%$, and $15.00 \%$ mortality in major workers. However, the mortality rates are $100.00 \%, 93.33 \%$, and $83.33 \%$ for the minors as well as $93.33 \%, 83.33 \%$, and $83.33 \%$ for the majors at $9 \mathrm{~d}$ of treatment.

\section{Grasping, walking, and aggregation rate}

Compared with the mortality of fumigation toxicity, the grasping, walking, and aggregation rates of RIFAs caused by volatiles from mashed leaves of $M$. exotica decrease over time (Figs 2, 3, and 4). At exposure times ranging from $1 \mathrm{~d}$ to $9 \mathrm{~d}$, the grasping abilities decrease from $86.7 \%$ to $6.7 \%$ (fresh 
leaves), $93.3 \%$ to $16.7 \%$ (fallen leaves), and $96.7 \%$ to $40.0 \%$ (dried leaves) for the major workers and from $95.0 \%$ to $0.0 \%$ (fresh leaves), $88.3 \%$ to $6.7 \%$ (fallen leaves), and $85.0 \%$ to $16.7 \%$ (dried leaves) for the minor workers (Fig 2, Table 3); the walking abilities are reduced from $86.7 \%$ to $6.7 \%$ (fresh leaves), $93.3 \%$ to $16.7 \%$ (fallen leaves), and $90.0 \%$ to $40.0 \%$ (dried leaves) for the major workers, as well as from $95.0 \%$ to
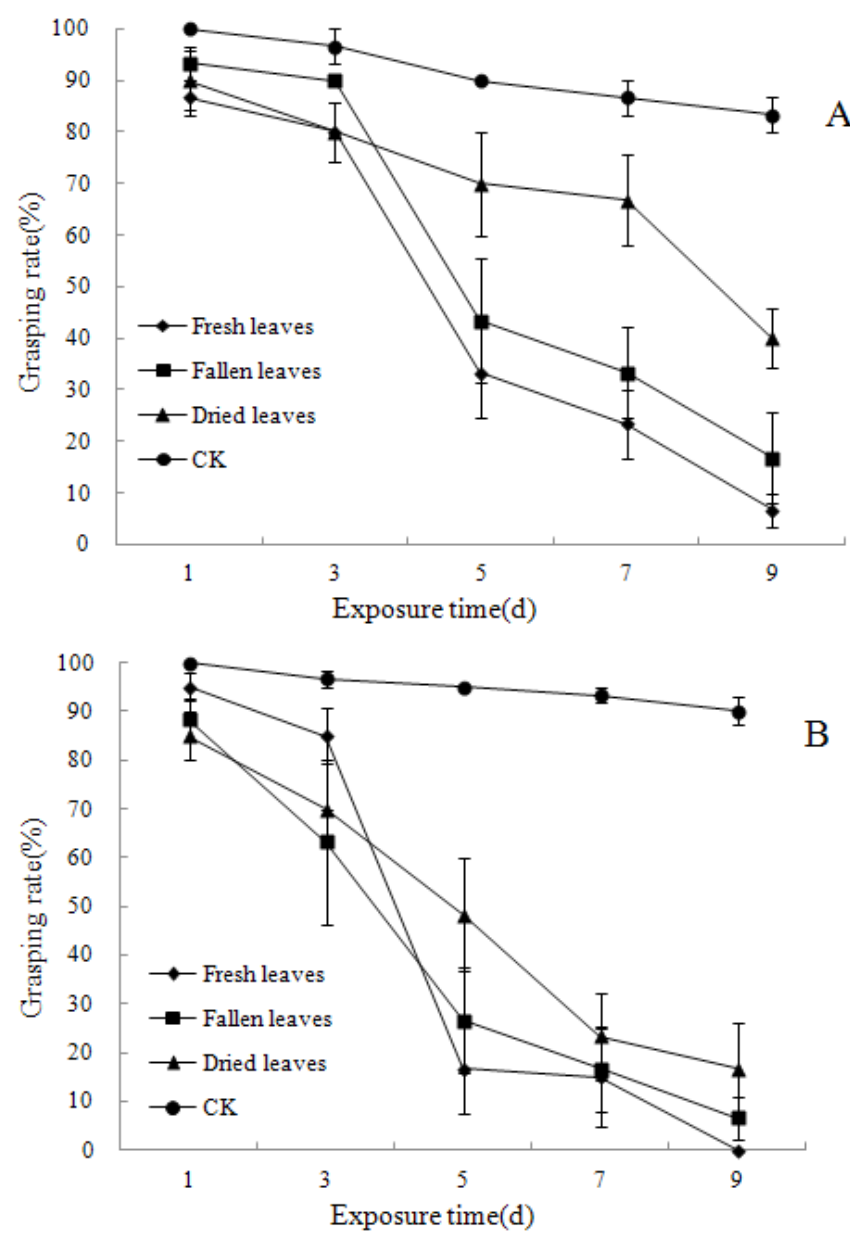

Fig 2. Effects on grasping ability of workers (A:major workers, B: Minor workers) after treated with different mashed leaves of $M$. exotica.
$0.0 \%$ (fresh leaves), $88.3 \%$ to $6.7 \%$ (fallen leaves), and $85.0 \%$ to $16.7 \%$ (dried leaves) for the minor workers (Fig 3, Table 4); the aggregation rate is reduced from $50.0 \%$ to $0.0 \%$ (fresh leaves), $86.7 \%$ to $16.7 \%$ (fallen leaves), and $50.0 \%$ to $40.0 \%$ (dried leaves) for the major workers and from $31.7 \%$ to $0.0 \%$ (fresh leaves), $68.3 \%$ to $3.3 \%$ (fallen leaves), and $51.7 \%$ to $13.3 \%$ (dried leaves) for the minor workers (Fig 4, Table5).
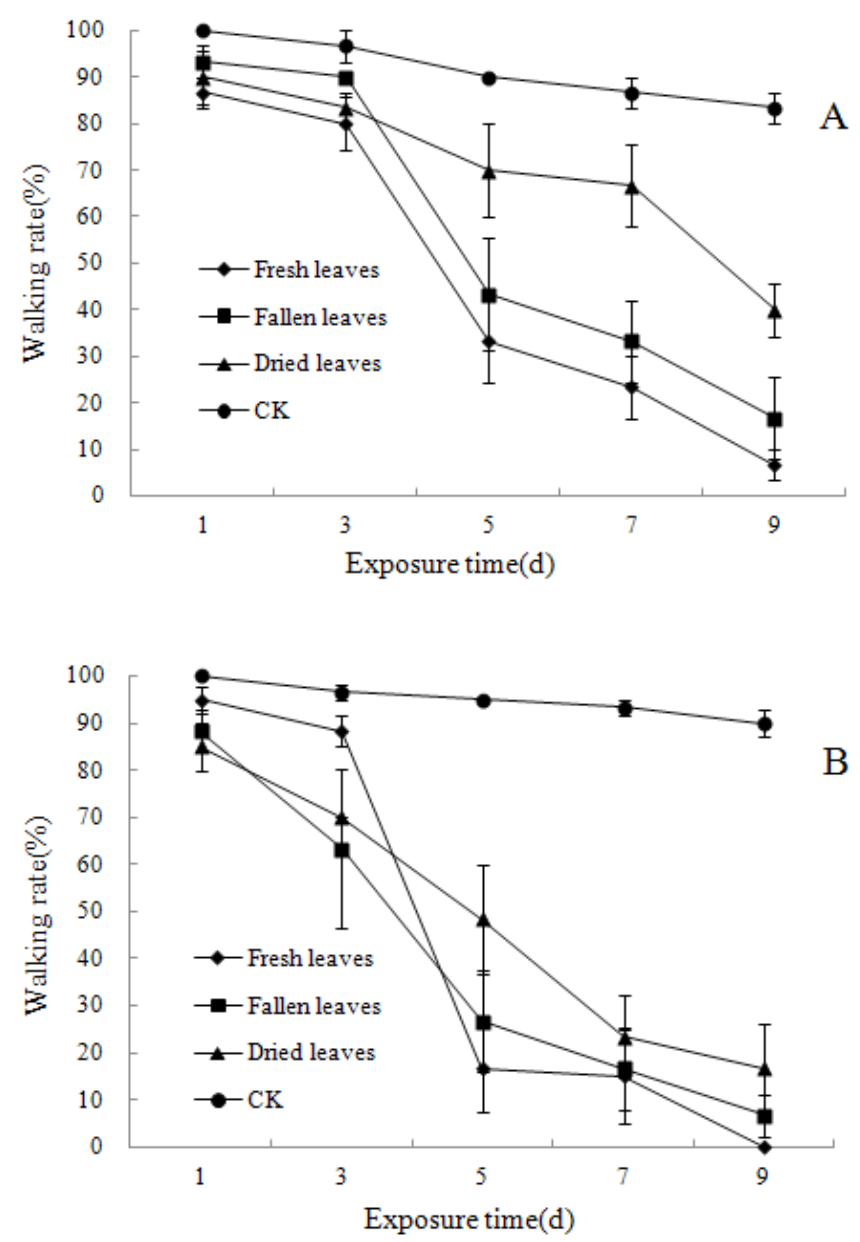

Fig 3. Effects on walking ability of workers (A: major workers, B: minor workers) after treated with different mashed leaves of $M$. exotica.

Table 2. Mortality of workers caused by mashed fresh, fallen, and dried leaves of M. exotica in the fumigation bioassay.

\begin{tabular}{|c|c|c|c|c|c|c|}
\hline Leaves & Workers & \multicolumn{5}{|c|}{ Mortality $(\%$, mean \pm SE) } \\
\hline Fresh & major & $13.3 \pm 3.3$ & $20.0 \pm 5.8$ & $66.7 \pm 8.8$ & $76.7 \pm 6.7$ & $93.3 \pm 3.3$ \\
\hline leaves & minor & $5.0 \pm 2.9$ & $11.7 \pm 3.3$ & $83.3 \pm 9.3$ & $85.0 \pm 10.0$ & $100.0 \pm 0.0$ \\
\hline Fallen & major & $6.7 \pm 3.3$ & $10.0 \pm 0.0$ & $56.7 \pm 12.0$ & $66.7 \pm 8.8$ & $83.3 \pm 8.8$ \\
\hline Dried & major & $10.0 \pm 3.3$ & $16.7 \pm 3.3$ & $30.0 \pm 10.0$ & $33.3 \pm 8.8$ & $60.0 \pm 5.8$ \\
\hline leaves & minor & $15.0 \pm 5.0$ & $30.0 \pm 0.0$ & $51.7 \pm 11.7$ & $76.7 \pm 8.8$ & $83.3 \pm 9.3$ \\
\hline \multirow{2}{*}{ ck } & major & $0.0 \pm 0.0$ & $3.3 \pm 3.3$ & $10.0 \pm 0.0$ & $13.3 \pm 3.3$ & $16.7 \pm 3.3$ \\
\hline & minor & $0.0 \pm 0.0$ & $3.3 \pm 1.7$ & $5.0 \pm 0.0$ & $6.7 \pm 2.9$ & $10.0 \pm 2.9$ \\
\hline
\end{tabular}


Table 3. Effects on grasping rate of workers after treated with mashed fresh, fallen, and dried leaves of M. exotica.

\begin{tabular}{|c|c|c|c|c|c|c|}
\hline \multirow{2}{*}{ Leaves } & \multirow{2}{*}{ Workers } & \multicolumn{5}{|c|}{ Grasping rate $(\%$, mean $\pm \mathrm{SE})$} \\
\hline & & $1 d$ & $3 d$ & $5 d$ & $7 d$ & $9 d$ \\
\hline Fresh & major & $86.7 \pm 3.3$ & $80.0 \pm 5.8$ & $33.3 \pm 8.8$ & $23.3 \pm 6.7$ & $6.7 \pm 3.3$ \\
\hline leaves & minor & $95.0 \pm 2.9$ & $85.0 \pm 5.8$ & $16.7 \pm 9.3$ & $15.0 \pm 10.0$ & $0.0 \pm 0.0$ \\
\hline Fallen & major & $93.3 \pm 3.3$ & $90.0 \pm 0.0$ & $43.3 \pm 12.0$ & $33.3 \pm 8.8$ & $16.7 \pm 8.8$ \\
\hline leaves & minor & $88.3 \pm 4.4$ & $63.3 \pm 16.9$ & $26.7 \pm 10.9$ & $16.7 \pm 8.8$ & $6.7 \pm 4.4$ \\
\hline Dried & major & $90.0 \pm 5.8$ & $80.0 \pm 5.8$ & $70.0 \pm 10.0$ & $66.7 \pm 8.8$ & $40.0 \pm 5.8$ \\
\hline leaves & minor & $85.0 \pm 5.0$ & $70.0 \pm 0.0$ & $48.3 \pm 11.7$ & $23.3 \pm 8.8$ & $16.7 \pm 9.3$ \\
\hline \multirow{2}{*}{ ck } & major & $100.0 \pm 0.0$ & $96.7 \pm 3.3$ & $90.0 \pm 0.0$ & $86.7 \pm 3.3$ & $83.3 \pm 3.3$ \\
\hline & minor & $100.0 \pm 0.0$ & $96.7 \pm 1.7$ & $95.0 \pm 0.0$ & $93.3 \pm 1.7$ & $90.0 \pm 2.9$ \\
\hline
\end{tabular}

Table 4. Effects on walking rate of workers after treated with mashed fresh, fallen, and dried leaves of M. exotica.

\begin{tabular}{|c|c|c|c|c|c|c|}
\hline \multirow{2}{*}{ Leaves } & \multirow{2}{*}{ Workers } & \multicolumn{5}{|c|}{ Walking rate $(\%$, mean \pm SE $)$} \\
\hline & & $1 \mathrm{~d}$ & $3 d$ & $5 d$ & $7 d$ & $9 d$ \\
\hline Fresh & major & $86.7 \pm 3.3$ & $80.0 \pm 5.8$ & $33.3 \pm 8.8$ & $23.3 \pm 6.7$ & $6.7 \pm 3.3$ \\
\hline Fallen & major & $93.3 \pm 3.3$ & $90.0 \pm 0.0$ & $43.3 \pm 12.0$ & $33.3 \pm 8.8$ & $16.7 \pm 8.8$ \\
\hline leaves & minor & $88.3 \pm 4.4$ & $63.3 \pm 16.9$ & $26.7 \pm 10.9$ & $16.7 \pm 8.8$ & $6.7 \pm 4.4$ \\
\hline leaves & minor & $85.0 \pm 5.0$ & $70.0 \pm 0.0$ & $48.3 \pm 11.7$ & $23.3 \pm 8.8$ & $16.7 \pm 9.3$ \\
\hline \multirow{2}{*}{$\mathrm{ck}$} & major & $100.0 \pm 0.0$ & $96.7 \pm 3.3$ & $90.0 \pm 0.0$ & $86.7 \pm 3.3$ & $83.3 \pm 3.3$ \\
\hline & minor & $100.0 \pm 0.0$ & $96.7 \pm 1.7$ & $95.0 \pm 0.0$ & $93.3 \pm 1.7$ & $90.0 \pm 2.9$ \\
\hline
\end{tabular}
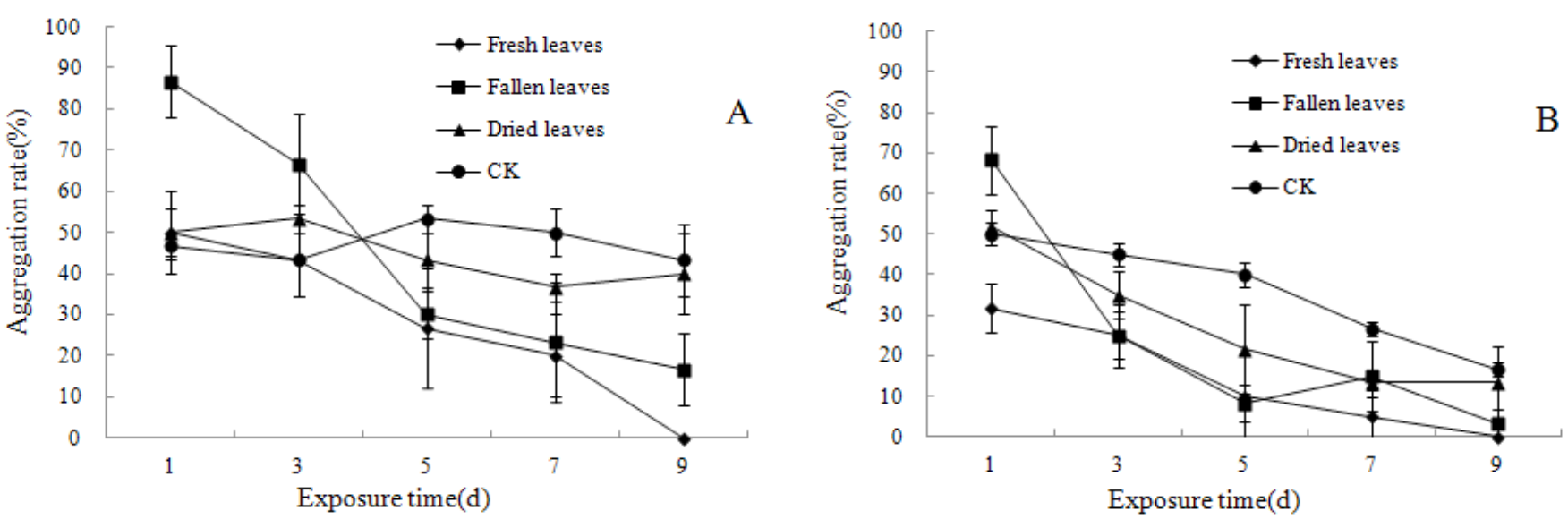

Fig. 4 Effects on aggregation of workers (A: major workers, B: minor workers) after treated with different mashed leaves of $M$. exotica.

\section{Chemical compositions of mashed leaves of Murraya exotica}

Figure 5 shows the GC-MS total ion chromatograms of extracts of fresh, fallen, and dried leaves of $M$. exotica. The result demonstrates that the volatile compounds from the mashed fresh, fallen, and dried of $M$. exotica contain 20, 28, and 21 major constituents, respectively (Tables 6, 7, and 8 ). The major components of fresh leaves comprising $89.82 \%$ of the total volatile compound are identified as $\beta$-caryophyllene $(40.20 \%),(+)-\beta$-himachalene $(17.76 \%)$, linalool (8.81\%), 1,3-cyclohexadiene, 5-(1,5-dimethyl-4hexenyl)-2-methyl, [S-( $\left.\left.\mathrm{R}^{*}, \mathrm{~S}^{*}\right)\right]-(7.00 \%)$, germacrene D
$(6.28 \%),(E)-\beta$-farnesene $(3.67 \%)$, a-copaene $(3.09 \%)$, and humulene $(3.01 \%)$. For fallen leaves, the $67.31 \%$ major components of the total volatile include $\beta$-caryophyllene (25.30\%), $\alpha$-cedrene (14.01\%), curcumene $(10.56 \%)$, trans- $\alpha$-bergamotene $(9.06 \%)$, germacrene D $(6.90 \%)$, (1R)-(+)- $\alpha$-pinene $(5.00 \%)$, and $\alpha$-copaene $(3.38 \%)$. The major components of dried leaves comprising $77.14 \%$ of the total volatile compound are $\alpha$-cedrene $(25.05 \%)$, $\beta$-caryophyllene (23.43\%), germacrene D (9.13\%), $\beta$-Cubebene $(8.79 \%)$, benzene, 1-(1,5-dimethylhexyl)4-methyl-(7.56\%), trans- $\alpha$-bergamotene $(6.38 \%)$, and $\alpha$-copaene $(3.18 \%)$. 
Table 5. Effects on aggregation rate of workers after treated with mashed fresh, fallen, and dried leaves of M. exotica.

\begin{tabular}{|c|c|c|c|c|c|c|}
\hline \multirow{2}{*}{ Leaves } & \multirow{2}{*}{ Workers } & \multicolumn{5}{|c|}{ Aggregation rate $(\%$, mean \pm SE) } \\
\hline & & $1 d$ & $3 d$ & $5 d$ & $7 \mathrm{~d}$ & $9 \mathrm{~d}$ \\
\hline Fresh & major & $50.0 \pm 5.8$ & $43.3 \pm 8.8$ & $26.7 \pm 14.5$ & $20.0 \pm 10.0$ & $0.0 \pm 0.0$ \\
\hline Fallen & major & $86.7 \pm 8.8$ & $66.7 \pm 12.0$ & $30.0 \pm 5.8$ & $23.3 \pm 14.5$ & $16.7 \pm 8.8$ \\
\hline leaves & minor & $68.3 \pm 8.3$ & $25.0 \pm 7.6$ & $8.3 \pm 4.4$ & $15.0 \pm 8.7$ & $3.3 \pm 3.3$ \\
\hline \multirow{2}{*}{$\mathrm{ck}$} & major & 46. $7 \pm 3.3$ & $43.3 \pm 8.8$ & $53.3 \pm 3.3$ & $50.0 \pm 5.8$ & $43.3 \pm 8.8$ \\
\hline & minor & $50.0 \pm 2.9$ & $45.0 \pm 2.9$ & $40.0 \pm 2.9$ & $26.7 \pm 1.7$ & $16.7 \pm 1.7$ \\
\hline
\end{tabular}

Abundance

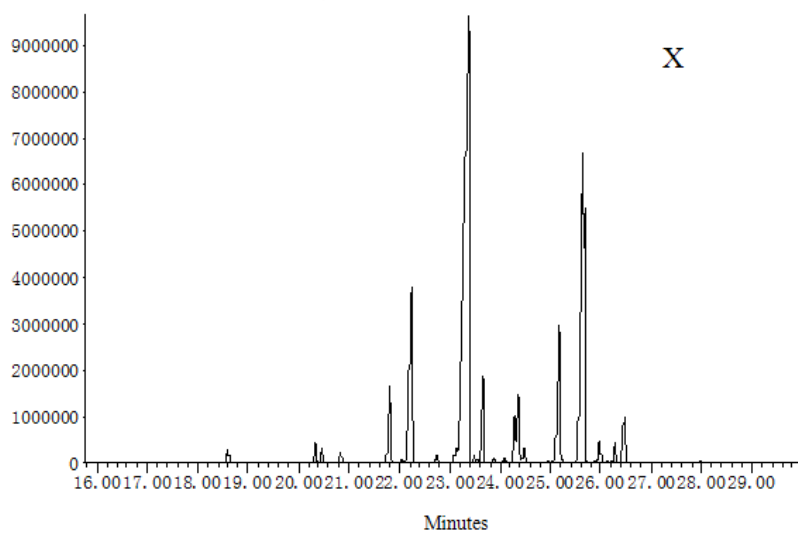

Abundance
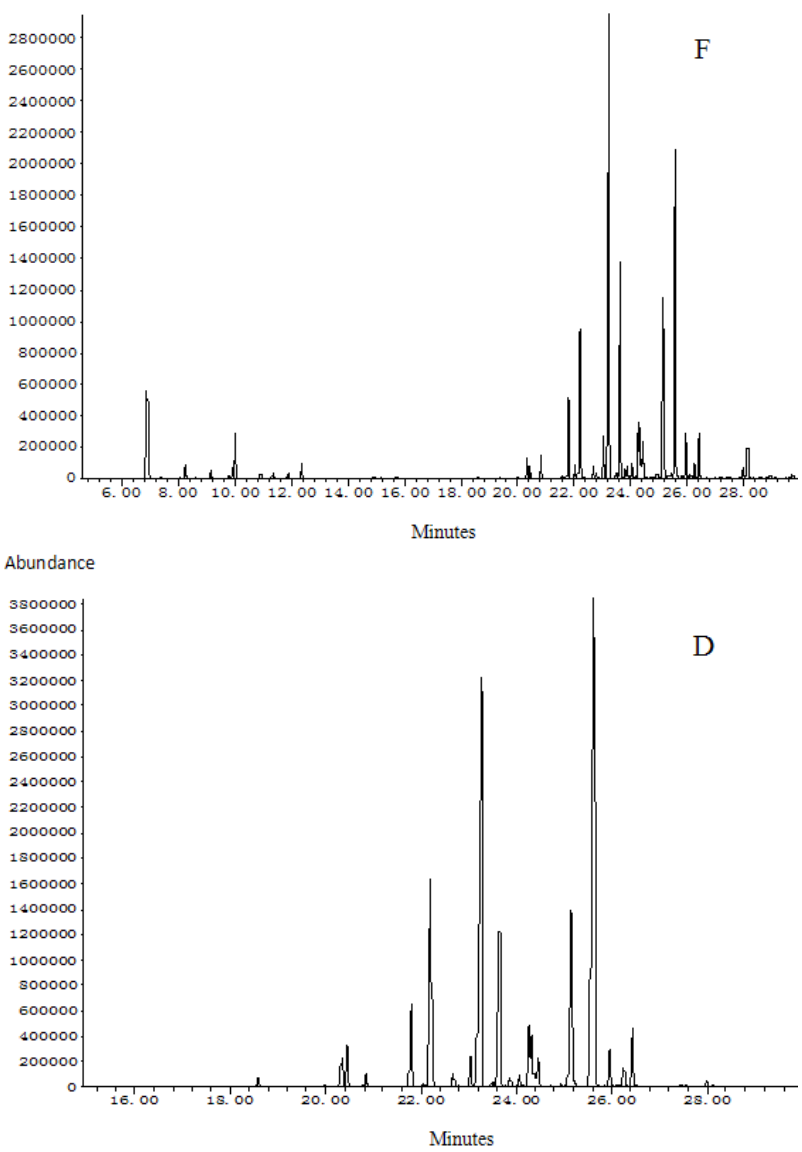

Fig. 5 GC-MS total ion chromatograms of mashed fresh(X), fallen(F), and dried(D) leaves of M. exotica.
Table 6. Chemical compositions from mashed fresh leaves of $M$. exotica.

\begin{tabular}{|c|c|c|c|}
\hline Number & Composition & $\begin{array}{c}\text { Relative retention } \\
\text { time }(\mathrm{min})\end{array}$ & $\begin{array}{c}\text { Percentage } \\
(\%)\end{array}$ \\
\hline 1 & Artemisia triene & 20.343 & 0.76 \\
\hline 2 & $\begin{array}{l}\text { 4-ethenyl-4-meth- } \\
\text { yl-1-(propan-2-yl)- } \\
\text { 3-(prop-1-en-2-yl) } \\
\text { cyclohexene }\end{array}$ & 20.454 & 0.57 \\
\hline 3 & $\alpha$-cubebene & 20.842 & 0.36 \\
\hline 4 & $\alpha$-copaene & 21.810 & 3.09 \\
\hline 5 & $(-)-\beta$-bourbonene & 22.050 & 0.18 \\
\hline 6 & Linalool & 22.239 & 8.81 \\
\hline 7 & $(\mathrm{Z}, \mathrm{E})$ - $\alpha$-farnesene & 22.742 & 0.41 \\
\hline 8 & $\begin{array}{l}\text { Bicyclo[3.1.1] } \\
\text { hept-2-ene,2,6-di- } \\
\text { metyl-6-(4-methyl- } \\
\text { 3-pentenyl)- }\end{array}$ & 23.125 & 0.66 \\
\hline 9 & $\beta$-caryophyllene & 23.381 & 40.20 \\
\hline 10 & (E)- $\beta$-farnesene & 23.665 & 3.67 \\
\hline 11 & $(-)-\beta$-santalene & 24.086 & 0.27 \\
\hline 12 & Humulene & 24.370 & 3.01 \\
\hline 13 & Alloaromadendrene & 24.477 & 0.60 \\
\hline 14 & $\beta$-copaene & 24.951 & 0.09 \\
\hline 15 & Germacrene D & 25.170 & 6.28 \\
\hline 16 & $(+)$ - $\beta$-himachalene & 25.640 & 17.76 \\
\hline 17 & $\begin{array}{l}\text { 1,3-Cyclohexa- } \\
\text { diene,5-(1,5-di- } \\
\text { methyl-4-hex- } \\
\text { enyl)-2-methyl-, } \\
{\left[\mathrm{S}-\left(\mathrm{R}^{*}, \mathrm{~S}^{*}\right)\right]-}\end{array}$ & 25.689 & 7.00 \\
\hline 18 & $\beta$-bisabolene & 25.978 & 0.88 \\
\hline 19 & d-cadinene & 26.270 & 0.72 \\
\hline 20 & $\begin{array}{l}\text { Cyclohexen- } \\
\text { e,3-(1,5-dimeth- } \\
\text { yl-4-hexen- } \\
\text { yl)-6-methylene-, } \\
{\left[\mathrm{S}-\left(\mathrm{R}^{*}, \mathrm{~S}^{*}\right)\right]-}\end{array}$ & 26.464 & 1.73 \\
\hline
\end{tabular}


Table 7. Chemical compositions from mashed fallen leaves of $M$. exotica.

\begin{tabular}{|c|c|c|c|}
\hline Number & Composition & $\begin{array}{c}\text { Relative retention } \\
\text { time }(\mathrm{min})\end{array}$ & $\begin{array}{c}\text { Percentage } \\
(\%)\end{array}$ \\
\hline 1 & $(1 \mathrm{R})-(+)-\alpha$-pinene & 6.880 & 5.00 \\
\hline 2 & $\beta$-pinene & 8.216 & 0.72 \\
\hline 3 & $\alpha$-phellandrene & 9.135 & 0.36 \\
\hline 4 & D-limonene & 9.898 & 0.49 \\
\hline 5 & Eucalyptol & 10.005 & 2.05 \\
\hline 6 & Linalool & 12.350 & 0.61 \\
\hline 7 & Artemisia triene & 20.330 & 0.89 \\
\hline 8 & $\begin{array}{l}\text { 4-ethenyl-4-meth- } \\
\text { yl-1-(propan-2-yl)- } \\
\text { 3-(prop-1-en-2-yl) } \\
\text { cyclohexene }\end{array}$ & 20.442 & 0.48 \\
\hline 9 & $\alpha$-cubebene & 20.829 & 0.89 \\
\hline 10 & $\alpha$-copaene & 21.781 & 3.38 \\
\hline 11 & $(-)-\beta$-bourbonene & 22.033 & 0.52 \\
\hline 12 & Germacrene D & 22.185 & 6.90 \\
\hline 13 & $\begin{array}{l}\text { Bicyclo[3.1.1] } \\
\text { hept-2-ene,2,6-di- } \\
\text { methyl-6-(4-methyl- } \\
\text { 3-pentenyl)- }\end{array}$ & 22.668 & 0.53 \\
\hline 14 & $(\mathrm{Z}, \mathrm{E})-\alpha$-farnesene & 23.014 & 1.69 \\
\hline 15 & $\beta$-caryophyllene & 23.241 & 25.30 \\
\hline 16 & trans- $\alpha$-bergamotene & 23.636 & 9.06 \\
\hline 17 & Alloaromadendrene & 23.768 & 0.42 \\
\hline 18 & $(-)-\beta$-santalene & 24.057 & 0.76 \\
\hline 19 & (E)- $\beta$-farnesene & 24.255 & 2.61 \\
\hline 20 & Humulene & 24.321 & 2.11 \\
\hline 21 & Aromadendrene & 24.448 & 1.64 \\
\hline 22 & Curcumene & 25.149 & 10.56 \\
\hline 23 & $\alpha$-cedrene & 25.574 & 14.01 \\
\hline 24 & 1- $\beta$-bisabolene & 25.949 & 1.83 \\
\hline 25 & d-cadinene & 26.246 & 0.63 \\
\hline 26 & $\begin{array}{l}\text { Cyclohexen- } \\
\text { e,3-(1,5-di- } \\
\text { methyl-4-hex } \\
\text { enyl)-6-methylene-, } \\
{\left[\mathrm{S}-\left(\mathrm{R}^{*}, \mathrm{~S}^{*}\right)\right]-}\end{array}$ & 26.427 & 2.02 \\
\hline 27 & Espatulenol & 27.981 & 0.50 \\
\hline 28 & $\begin{array}{l}\text { Caryophyllene } \\
\text { oxide }\end{array}$ & 28.133 & 1.50 \\
\hline
\end{tabular}

\section{Discussion}

Our results have shown that the volatile compounds released from the mashed fresh, fallen, and dried leaves of $M$. exotica evidently reduce the grasping and walking abilities and aggregation rate of the RIFA workers. Furthermore, volatile compounds of the leaves of $M$. exotica have been showed to be insecticidal.

Plant insecticides are relatively safe, degradable, and readily available in many regions of the world; hence, these
Table 8. Chemical compositions from mashed dried leaves of $M$. exotica.

\begin{tabular}{|c|c|c|c|}
\hline Number & Composition & $\begin{array}{l}\text { Relative retention } \\
\text { time (min) }\end{array}$ & $\begin{array}{c}\text { Percentage } \\
(\%)\end{array}$ \\
\hline 1 & Camphene & 20.339 & 1.11 \\
\hline 2 & $\begin{array}{l}\text { 4-ethenyl-4-meth- } \\
\text { yl-1-(propan-2-yl)- } \\
\text { 3-(prop-1-en-2-yl) } \\
\text { cyclohexene }\end{array}$ & 20.458 & 1.59 \\
\hline 3 & $\alpha$-cubebene & 20.838 & 0.47 \\
\hline 4 & $\alpha$-copaene & 21.794 & 3.18 \\
\hline 5 & Germacrene D & 22.210 & 9.13 \\
\hline 6 & $(\mathrm{Z}, \mathrm{E})$ - $\alpha$-farnesene & 22.684 & 0.50 \\
\hline 7 & $\begin{array}{l}\text { Bicyclo[3.1.1] } \\
\text { hept-2-ene,2,6-di- } \\
\text { metyl-6-(4-methyl- } \\
\text { 3-pentenyl)- }\end{array}$ & 23.039 & 1.22 \\
\hline 8 & $\beta$-caryophyllene & 23.278 & 23.43 \\
\hline 9 & trans- $\alpha$-bergamotene & 23.649 & 6.38 \\
\hline 10 & cis- $\beta$-farnesene & 23.871 & 0.38 \\
\hline 11 & epi- $\beta$-santalene & 24.073 & 0.51 \\
\hline 12 & $\begin{array}{l}\text { 1,3-Cyclohexa- } \\
\text { diene,5-(1,5-di- } \\
\text { methyl-4-hex- } \\
\text { enyl)-2-methyl-, } \\
{\left[\mathrm{S}-\left(\mathrm{R}^{*}, \mathrm{~S}^{*}\right)\right]-}\end{array}$ & 24.271 & 2.59 \\
\hline 13 & Humulene & 24.337 & 1.97 \\
\hline 14 & Alloaromadendrene & 24.461 & 1.12 \\
\hline 15 & $\beta$-cubebene & 25.145 & 8.79 \\
\hline 16 & $\alpha$-cedrene & 25.619 & 25.05 \\
\hline 17 & $\begin{array}{l}\text { Benzene, 1-(1,5-di- } \\
\text { methylhexyl)-4-m } \\
\text { ethyl- }\end{array}$ & 25.648 & 7.56 \\
\hline 18 & $\beta$-bisabolene & 25.969 & 1.39 \\
\hline 19 & d-cadinene & 26.258 & 0.73 \\
\hline 20 & $\begin{array}{l}\text { Cyclohexen- } \\
\text { e,3-(1,5-dimeth- } \\
\text { yl-4-hexen- } \\
\text { yl)-6-methylene-, } \\
{\left[S-\left(R^{*}, S^{*}\right)\right]-}\end{array}$ & 26.452 & 2.26 \\
\hline 21 & $\alpha$-guaiene & 27.985 & 0.25 \\
\hline
\end{tabular}

insecticides could replace some traditional chemicals. Li et al. (2014) reported that volatile compounds of Tephrosia vogelii exhibited high toxicity against the RIFA workers. The essential oils of M. exotica possessed fumigant toxicity against Sitophilius zeamais, and Tribolium castaneum adults with $\mathrm{LC}_{50}$ values of 8.29 and $6.84 \mathrm{mg} / \mathrm{L}$, respectively. According Govindarajan et al. (2012), the essential oils from Mentha spicata (Linn.) exhibited larvicidal activity against three mosquito species, Aedes aegypti, Anopheles stephensi, and Culex quinquefasciatus. The natural product of the leaves of Boenninghausenia albiflora was active against Plecoptera reflexa, Clostera cupreata, and Crypsiptya coclesalis at different concentrations varying from $1.0 \%$ to $5 \% \mathrm{w} / \mathrm{v}$ (Sharma et al. 2006). The acetone extract of $M$. exotica leaves 
showed an antifeedant activity against the early third-stage larvae of Spodoptera litura (Wang et al. 2009).

The results of the fumigation toxicity bioassay have shown that the fresh leaves of $M$. exotica is more active against RIFAs than fallen and dried leaves. The volatile compounds of mashed leaves of $M$. exotica are $\beta$-caryophyllene, $\alpha$-cedrene, $\alpha$-copaene, $\beta$-cubebene, and germacrene $\mathrm{D}$. This finding is similar to that reported by Jiang et al. (2009). According to the results of $\mathrm{GC}-\mathrm{MS}, \beta$-caryophyllene is the major component of the leaves of $M$. exotica. The content of $\beta$-caryophyllene is highest in fresh leaves $(40.20 \%)$ among in the fallen $(25.30 \%)$ and in the dried leaves $(23.43 \%)$. These observations show that the significant activity of $M$. exotica leaf may be due to the presence of major chemical constituents such as $\beta$-caryophyllene. Several studies reported that $\beta$-caryophyllene in other plants possessed insecticidal activity (Zhu \& Tian, 2011; Krishnamoorthy et al., 2015; Venturi et al., 2015; Salleh et al., 2015). M. exotica as a popular hedge plant well adapted for topiary work is widely distributed in southern China and several tropical and subtropical regions of Asia. In these M. exotica growing areas, the plant need to trim every year and there are a lot of fresh leaves cut from it. Therefore the fresh leaves cut from $M$. exotica can be crushed thoroughly and used to control RIFAs.

The volatile compounds from the mashed fresh of $M$. exotica possess high insecticide activity against the RIFA workers. These facts indicate that the leaves of $M$. exotica have potential for controlling these pest ants. Fresh leaves of M. exotica could be collected and used as raw materials to control RIFA.

\section{Acknowledgment}

This study were funded by the talent development program on joint training of postgraduates demonstration base in Guangdong Province, China (2013JDXM11), science and technology planning project of Guangdong Province (2014A020208114) and science and technology planning and scientific research project of Guangzhou city (201510010299).

\section{References}

Appel, A. G., Gehret, M. J. \& Tanley, M. J. (2004). Repellency and toxicity of mint oil granules to red imported fire ants (Hymenoptera: Formicidae). Journal of Economic Entomology, 97: 575-580. doi: 10.1603/0022-0493-97.2.575

Ascunce, M. S., Yang, C. C., Oakey J., Calcaterra, L., Wu, W. J., Shih, C. J., Goudet, J., Ross, K. G. \& Shoemaker, D. (2011). Global invasion history of the fire ant, Solenopsis invicta. Science, 331: 1066-1068. doi: 10.1126/science.1198734

Cheng, S. S., Liu, J. Y., Lin, C. Y., Shui, Y. R., Lu, M. C., Wu, W. J. \& Chang, S. T. (2008). Terminating red imported fire ants using Cinnamomum osmophloeum leaf essential oil. Bioresource Technology, 99: 889-893. doi: 10.1016/j. biortech.2007.01.039

Devigne C., Broly, P. \& Deneubourg, J. (2011). Individual Preferences and Social Interactions Determine the Aggregation of Woodlice. Plos One, 6(2): 563-565. doi: 10.1371/journal. pone.0017389

Depickère, S., Fresneau, D. \& Deneubourg, J. (2004). Dynamics of aggregation in Lasius niger (Formicidae): influence of polyethism. Insectes Sociaux, 51: 81-90. doi: 10.1007/s00040-003-0719-8

Govindarajan, M., Sivakumar, R., Rajeswari, M. \& Yogalakshmi, K. (2012). Chemical composition and larvicidal activity of essential oil from Mentha spicata (Linn.) against three mosquito species. Parasitology Research, 110: 20232032. doi:10.1007/s00436-011-2731-7

Krishnamoorthy, S., Chandrasekaran, M. \& Raj, G. (2015). Identification of chemical constituents and larvicidal activity of essential oil from Murraya exotica L. (Rutaceae) against Aedes aegypti, Anopheles stephensi and Culex quinquefasciatus (Diptera: Culicidae). Parasitology Research, 5: 1839-1845. doi:10.1007/s00436-015-4370-x

Li, H. X., Wei, M. S. \& Yi, P. Y. (2001). Effect of 25 plant essential oil on Callosobruchus maculates. Grain Storage, 6: 7-9. doi:10.3969/j.issn.1000-6958.2001.06.002

Luo, Y. P., Zhu, Z. H. \& Tan, Z. Q. (2005). Antifeedant activities of extracts of Murraya paniculata against Spodptera litura fabricius. Hubei Agricultural Sciences, 6: 49-51. doi: 10. 14088/j.cnki.issn0439-8114.2005.06.015

Li, W. S., Zhou, Y., Li, H., Chen, D. M. \& Zhang, Z. X. (2014). Insecticidal effect of volatile compounds from fresh plant materials of Tephrosia vogelii against Solenopsis invicta workers. Sociobiology, 61: 28-34. doi: 10.13102/sociobiology. v61i1.28-34

Li, W. Q., Jiang, C. H., Chu, S. S., Zuo, M. X. \& Liu, Z. L. (2010). Chemical Composition and Toxicity against Sitophilus zeamais and Tribolium castaneum of the Essential Oil of Murraya exotica Aerial Parts. Molecules, 15: 58315839. doi:10.3390/molecules 15085831.

Negi, N., Ochi, A., Kurosawa, M., Ushijima, K., Kitaguchi, Y., Kusakabe, E., Okasho, F., Kimachi, T., Teshima, N., Juichi, M., Abou-Douh, A. M., Ito, C. \& Furukawa, H. (2005). Two new dimeric coumarins isolated from Murraya exotica. Chemical and Pharmaceutical Bulletin, 53: 1180-1182. doi: 10.1002/chin.200608191

Nattrass, R., \& Vanderwoude, C. (2001). A preliminary investigation of the ecological effects of red imported fire ants (Solenopsis invicta) in Brisbane. Ecological Management and Restoration, 2: 220-223.

Olawore, N. O., Ogunwander, I. A., Ekundayo, O. \& 
Adeleke, K. A. (2005). Chemical composition of the leaf and fruit essential oils of Murraya paniculata (L.) Jack. (Syn. Murraya exotica Linn.). Flavour and Fragrance Journal, 20: 54-56. doi: 10.1002/ffj.1365

Pino, J. A., R. Marbot, \& V. Fuentes. (2006). Aromatic plants from western Cuba. VI. Composition of the leaf oils of Murraya exotica L., Amyris balsamifera L., Severinia buxifolia (Poir.) Ten. and Triphasia trifolia (Burm. f.) P. Wilson. Journal of Essential Oil Research, 18: 24-28. doi: 10. 1080/10412905.2006.9699376

Porter, S. D. \& Savignano, D. A. (1990). Invasion of polygyne fire ants decimates native ants and disrupts arthropod community. Ecology, 71: 2095-2106. doi:10.2307/1938623

Rout, P. K., Rao, Y. R., Sree, A. \& Naik, S. N. (2007). Composition of essential oil, concrete, absolute, wax and headspace volatiles of Murraya paniculata (Linn.) Jack flowers. Flavour and Fragrance Journal, 22: 352-357. doi: 10.1002/ffj.1804

Raina, V. K., Verma, S. C., Dhawan, S., Khan, M., Ramesh, S., Singh, S. C., Yadav, A. \& Srivastava, S. K. (2006). Essential oil composition of Murraya exotica from the plains of northern India. Flavour and Fragrance Journal, 21: 140142. doi: 10.1002/ffj.1547

Sharma, R., Negi, D. S. \& Shin, W.K.P. (2006). Characterization of an insecticidal coumarin from Boenninghausenia albiflora. Phytotherapy Research, 7: 607-609. doi: 10.1002/ptr.1909

Venturi, C. R., Danielli, L. J. \& Klein, F. (2015). Chemical analysis and in vitro antiviral and antifungal activities of essential oils from Glechon spathulata and Glechon marifolia. Pharmaceutical Biology, 5: 682-688. doi: 10.3109/13880209.2014.936944

Wang, L. Y., Lu, Y. Q. \& Luo, Y. P. (2009). Antifeeding activities of 45 south herbsextracts against Spodpteralitura Fabricius. Hubei Agricultural Science, 48: 628-630. doi:10.14088/j.cnki.issn0439-8114.2009.03.025

Wan, S. W. M. N. H. W., Ahmad, F. \& Yen, K. H. (2015). Chemical compositions and biological activities of the essential oils of Beilschmiedia madang Blume (Lauraceae). Archives of Pharmacal Research, 4: 485-493. doi: 10.1007/ s12272-014-0460-z

Yu, D. Z., Yang, X. J. \& Yang, L. J. (2001). Antibacterial activity of plant extracts against plant pathogenic fungi. Hubei Agricultural Sciences, 5: 49-51. doi: 10.14088/j.cnki. issn0439-8114.2001.05.021

Zhang, D. X., Hartley, T. G. \& Mabberley, D. J. Flora of China. http://foc.eflora.cn/cecontent.aspx?TaxonId=10781. (accessed online in 2008).

Zhu, L., \& Tian, Y. (2011). Chemical composition and larvicidal activity of Blumea densiflora essential oils against Anopheles anthropophagus:a malarial vector mosquito. Parasitology Research, 109: 1417-1422. doi: 10.1007/s00436-011-2388-2

Zhang, R., Li, Y. Liu, N. \& Porter, S. D. (2007). An Overview of the Red Imported Fire Ant (Hymenoptera: Formicidae) in Mainland China. Florida Entomologist, 90: 723-731. 